\title{
The Role of Differentially Expressed miRNAs and Potential miRNA-mRNA Regulatory Network in Prostate Cancer Progression and Metastasis
}

\author{
Elif Damla Arisan ${ }^{1}$, Ines Lua Freitas ${ }^{2}$, Kimia Pazoki², Sahra Rahim² and Pinar Uysal-Onganer ${ }^{2 *}$ \\ ${ }^{1}$ Institute of Biotechnology, Gebze-Kocaeli, Turkey \\ ${ }^{2}$ School of Life Sciences, College of Liberal Arts and Sciences, 115 New Cavendish Street, London W1W 6UW, UK
}

*Corresponding author: Pinar Uysal Onganer, School of Life Sciences, College of Liberal Arts and Sciences, 115 New Cavendish

Street, London W1W 6UW, UK

\section{ARTICLE INFO}

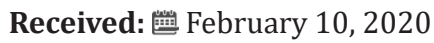

Published: February 18, 2020

Citation: Elif Damla A, Ines Lua F, Kimia P, Sahra R, P Uysal-Onganer. The Role of Differentially Expressed miRNAs and Potential miRNA-mRNA Regulatory Network in Prostate Cancer Progression and Metastasis. Biomed J Sci \& Tech Res 25(4)-2020. BJSTR. MS.ID.004241.

Abbreviations: PCa: Prostate Cancer; TGF- $\beta$ : Transforming Growth Factor Beta; CAMs: Cell Adhesion Molecules; EGF: Epidermal Growth Factor; MAPK: MitogenActivated Protein Kinases; RTK: Receptor Tyrosine Kinase; PTEN: Phosphatase and Tensing Homolog; ATCC: American Type Culture Collection; FBS: Foetal Bovine Serum; FCS: Foetal Calf Serum; PDCD4: Programmed Cell Death 4; GO: Gene Ontology; INVL: Invasion Index; FAK: Focal Adhesion Kinase; KEGG: Kyoto Encyclopedia Of Genes And Genomes

\begin{abstract}
Purpose: Aberrant expression of microRNAs (miRNAs) has been discovered in prostate cancer progression however their function is not well understood, thereby further investigation is required to understand the importance of underlying mechanisms and their involvement in multiple signaling pathways, as well as their potential as therapeutic targets. In this study the role and expression levels of three miRNAs were evaluated: miR-21, miR-221 and miR-200c in different prostate cancer cell lines. In addition, based on the latest studies on miRNAs function, their association with other target genes and molecules were analyzed using bioinformatic tools.
\end{abstract}

Methods: Three PCa cell lines PC3, LNCaP and VCaP and normal prostate epithelial cell line PNT1A were screened for miRNA expression levels using qPCR. miRNA target genes and their association with signaling pathways were analyzed through several Network and pathway analysis online tools

Findings: Upregulation of miR-21 and miR-221 was observed in PC3 and VCaP prostate cancer cells, respectively. According to KEGG analysis, we found that Hippo signaling pathway and cytokine-cytokine receptor interactions were affected by miR-21 while miR-221 would interfere with ECM-receptor interaction, Fatty acid elongation and Huntington disease molecular networks. Exposure of PC3 cells to TGF- $\beta(10 \mu \mathrm{M})$ caused upregulation of miR-21 with the evidence with increased invasion potential.

Discussion and Conclusion: miRNAs could regulate several genes in multiple signaling pathways. Here, we demonstrated that in a panel of PCa cell lines, both mir-21 and miR-221 expressions were upregulated. miR-21 may be a diagnostic and prognostic biomarker for PCa.

Keyword: miRs, Prostate Cancer, Invasion, TGF- $\beta$, miR-21

\section{Introduction}

Prostate cancer (PCa) is the most common cancer in men and a major cause of cancer-related deaths worldwide [1] PCa often progresses from hormone sensitive to castrate resistance [2]. The rates of PCa mortalities increase each year due to recurrence and metastasis, where developing new biomarkers could lead to early diagnosis, prognosis and effective treatment. microRNAs (miRs) are endogenous small non-coding RNA molecules with around 22 nucleotides and are regulators of gene expression at the post transcriptional level that can affect mRNA translation [3]. Highly conserved and stable miRNAs are expressed uniquely across different types of human cancers including PCa [4]. miR-21 is amongst the most commonly studied miRNAs, acts as oncogene being called oncomiR, it is thought to be highly expressed in both androgen dependent and independent $\mathrm{PCa}[5,6]$. miR-221 however, is expressed together with miR-222 and its function can be both oncogenic and tumour suppressor depending on the tissue type 
[7]. It is unclear whether miR-221 regulates PCa progression from androgen dependence to castration resistance while acting as oncomiR. On the other hand, a member of the miR-200 family, miR-200c, is reported to be involved in the progression of various cancers as a tumour suppressor and inhibits PI3K-Akt pathway [8].

Transforming growth factor beta (TGF- $\beta$ ) activator of SMADs and superfamily of cytokines is a signaling process involved in cancer progression. TGF-B is known to be a target for one or several miRNAs, leading to changes in the cell behaviour and tumor microenvironment [9]. Both miR-21 and miR-200 are identified to be targeted by the components of TGF- $\beta$ pathway, and this association could lead to potential therapeutic targets for PCa [10]. Epithelial-mesenchymal transition (EMT), a process that changes epithelial cells into fibroblast looking cell types known as mesenchymal cells, accordingly, known to play an important role in morphological changes during embryonic development [11]. This is highly regulated process is identified to play a role in tumour metastasis; thereby EMT markers could be potential targets for cancer diagnosis and prognosis. E-cadherin, an epithelial marker from a family of cell adhesion molecules (CAMs) is one of the most important molecules encoded by $\mathrm{CDH} 1$ gene. It plays an important role as a tumour suppressor and found to be suppressed in many cancers such as PCa suggesting that it can be an important marker in cancer progression and metastasis [12].

Though epithelial markers are important biomarkers, mesenchymal markers such as $\mathrm{N}$-cadherin and Vimentin also play a crucial role in migration and metastasis and are often overexpressed in cancers. Other EMT markers such as matrix metalloproteinases (e.g. MMP2 and MMP9) are significant as they have the ability to breakdown collagen, an important structural component of basement membrane, in other words it is an essential factor in extracellular matrix (ECM) breakdown. MMP is also known to be highly associated with disease development and metastasis $[13,14]$ and miR-21 might alter MMP signalling [15]. EMT genes could be targeted by several miRNAs, which subsequently deregulate their function and expression levels. Epidermal growth factor (EGF) could induce RAS and mitogen-activated protein kinases (MAPK) signalling through receptor tyrosine kinase (RTK) activation, where downstream components of this pathway were studied in PCa. miRs could also alter PI3K/AKT/mTOR signalling axis, which is one of the central signalling pathways that is involved in cell proliferation, differentiation and cell survival [16].

An important regulator in the PI3K pathway known as phosphatase and tensing homolog (PTEN) is a tumour suppressor, targeted and regulated by miR-21, leading to its suppression in tumour cells. Many miRNAs are known to regulate signalling pathways and be regulated through several mRNAs within these cascades, either enhancing or suppressing tumorigenesis. Having all this info together, this study evaluates the influence of cytokine activity within several signalling cascades and how cross-talks between these pathways could lead to initiation of EMT and metastasis in PCa.

\section{Methods}

\section{Cell Lines and Cell Culture}

PCa cell lines PNT1A, LNCaP, PC3 and VCaP cells were purchased from the American Type Culture Collection (ATCC) and were maintained in RPMI-1640 (Invitrogen) and supplemented with $10 \%$ foetal bovine serum (FBS) with the addition of 1\% Penicillin/ Streptomycin, then incubated at $37^{\circ} \mathrm{C}, 5 \% \mathrm{CO} 2$.

\section{Total RNA Extraction, cDNA Synthesis and qtr.-PCR}

Total RNA was extracted by using Triazole (Sigma, UK) according to the manufacturer's instructions. The purity and concentration of the isolated RNA were measured using the Nanodrop spectrophotometer at $260 \mathrm{~nm}$ and $280 \mathrm{~nm}$ absorbance. For cDNA production, the script microRNA cDNA Synthesis Kit (Quanta bio, U.K.) was used according to the manufacturer's instructions. The cDNA was used to assess the expression of miR-21, miR-221 and miR-200c. U6-snRNA and hsa-let-7a-5p were used as reference RNAs for normalization of miR expression levels by using Perfecta SYBR Green Super Mix (Quanta bio, U.K.). Thermocycling conditions were used as described before [17]. $2 \Delta \Delta \mathrm{CT}$ method [18] was used for calculating relative Mir expression levels and for normalization. Each experiment was repeated three times.

\section{Cell Viability and Invasion Assays}

Cell invasiveness by assessed as described before [19]. PC-3 cells were trypsin zed after treating with TGF- $\beta 1$ (RnD System, U.K) alongside with the controls and plated at $5 \times 104$ on Matrigel coated inserts with $8 \mathrm{u}$ filters (BD Biosciences, U.K.) in growth medium containing $1 \%$ FCS. Cells then were stimulated to migrate across the filters by providing $10 \%$ foetal calf serum (FCS) as a chemo-attractant in the assay chambers beneath the inserts. After $16 \mathrm{~h}$, the number of invaded cells was determined by MTT assay and this was confirmed by crystal violet assay. In parallel cell number was detected by MTT assay 19 after 16h. Invasion was presented as "Invasion Index (InvI)" i.e. percentage (\%) of the readings for invaded cells/original cell number.

\section{Bioinformatics}

miRNA Prediction based tool Mir Path v.3 - DIANA TOOLS (http://snf- 515788.vm.okeanos.grnet.gr) was used to find miRNAs target genes discovered in signalling pathways (e.g. Wnt signalling, JAK/STAT signalling and EMT) through Kyoto encyclopedia of genes and genomes (KEGG) and gene ontology (GO) analysis (Li et al, 2018). KEGG Reverse search tool was used for more specific miRNA targets through pathway search tool (e.g. Wnt signalling pathway|hsa04310) using Tarbase v.7 method. miRmap (https:// mirmap.ezlab.org/app/) and miRDB (http://mirdb.org) were used to find target genes associated with miRNAs based on predictive analysis. Functional analysis of protein-protein interactions for 
3 genes (AR, CDH1 and PTEN) were created using STRING v.11 database tool analysis (https://string-db.org). Moreover, KEGG pathway viewer online tool (https://www.genome.jp/kegg/ pathway.html) was used to show critical PCa pathways targeted by miRNAs that were associated with tumour progression, cell proliferation, apoptosis and cell cycle events.

\section{Data Analysis}

The graphs and histograms were prepared, and statistical analysis was performed using GraphPad Prism version 6 (GraphPad Software, San Diego, U.S.A.). One-way ANOVA was performed followed by Tukey's post-hoc analysis; significant differences were considered as $\mathrm{p} \leq 0.05$.

\section{Results}

\section{MicroRNA Expression of PCa Cell Lines}

The expression levels of miR-21, miR-221 and miR-200c in different PCa cell lines were analysed and compared with PNT1A, normal epithelial prostate cells by RT-qPCR. PC3 cells have the highest level of miR-21 and miR-221 and the lowest expression of miR-200c (Figure 1, n=3; $\mathrm{p}<0.05$ overall). On the contrary, expression of miR-200c was detected in PNT1A and LNCaP cells but not in PC3 cells ( $n=3$ p>0.05 for PC3). Androgen sensitive metastatic VCaP cells expressed both miR-21 and miR-221 however, expression levels of both miRs were lower than PC3.

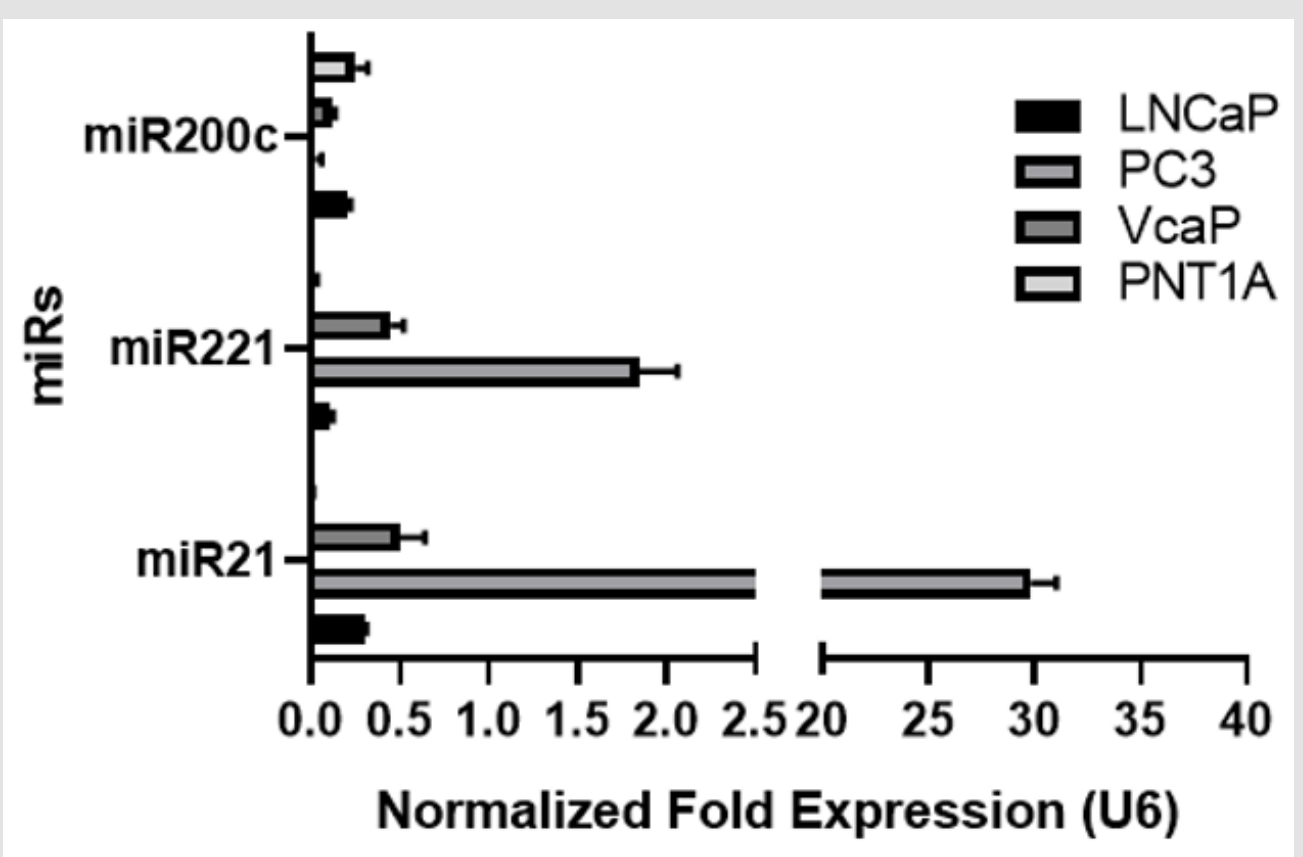

Figure 1: microRNA expression profile of PCa cell lines by RT-qPCR. Higher expression of miR-221 and miR-21 were detected in PC3 and VCaP cells; low expression of miR21 and 221 was found in PNT1A and LNCaP cell lines. MiR-200c was downregulated in VcaP and PC3 cell lines whereas PNT1A and LNCaP cells expressed higher level of miR-200c. Data is normalized to U6snRNA and hsa-let-7a. Graph represent three independent replicates.

\section{Molecular Signalling Pathways Affected By miR-200c,} MIR-221 AND MIR-21

We then run an in-silico experiment to identify molecular networks orchestrated by these miRs. According to KEGG analysis, we found that Hippo signalling pathway and cytokinecytokine receptor interactions were affected by miR-21 while miR-221 would interfere with ECM-receptor interaction, Fatty acid elongation and Huntington disease molecular networks. We did not detect any overlapping network hierarchy for any Mir targets (Figure 2A). Gene Ontology (GO) analysis showed that only organelle functions could be a similar target for miR-21 and miR221. In turn, GO analysis also showed that miR-21 expression levels significantly affect mechanisms as gene expression, nucleic acid binding transcription factor activity, neurotrophin TRK receptor signalling pathway, cellular nitrogen metabolic compound process and biosynthetic process (Figure 2B). Here, we concluded that highly metastatic PCa cells showed significant alteration levels on different cell functions due to high expression levels of miR-21. Additional upregulation of miR-221 exerted additive alterations on cell hierarchy by disturbing organelle functions. 
A

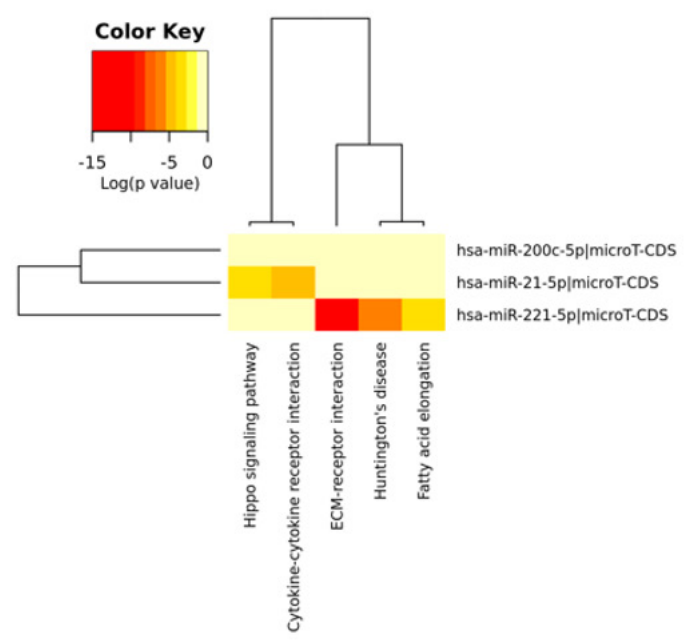

B
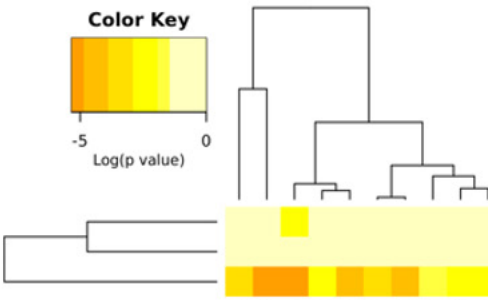

Figure 2: Signalling pathways and molecular mechanisms affected by miR-200c, miR-221 and miR-21.

A. KEGG analysis shows Hippo signalling pathway and cytokine-cytokine receptor interactions were affected by miR-21 and ECM-receptor interaction, Fatty acid elongation and Huntington disease molecular networks were controlled by miR-221. B. GO analysis demonstrated miR-21 regulates gene expression, nucleic acid binding transcription factor activity, neurotrophin TRK receptor signalling pathway, cellular nitrogen metabolic compound process and biosynthetic process.

\section{TGF-B Mediated Mir-21 Upregulation Triggered Invasion Potential of PC3 Prostate Cancer Cells}

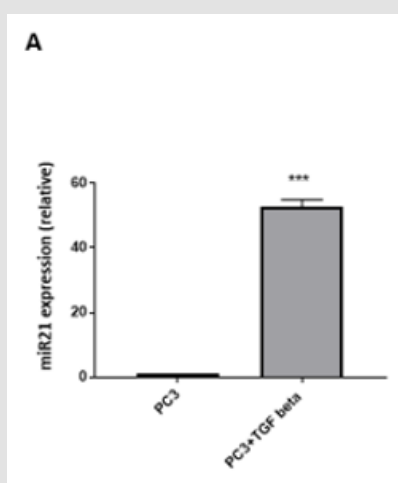

c

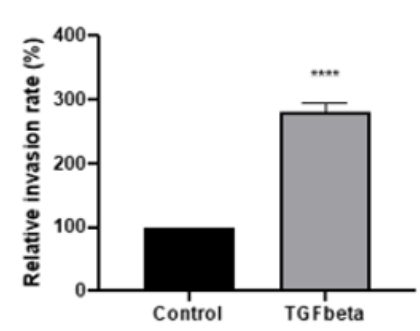

B
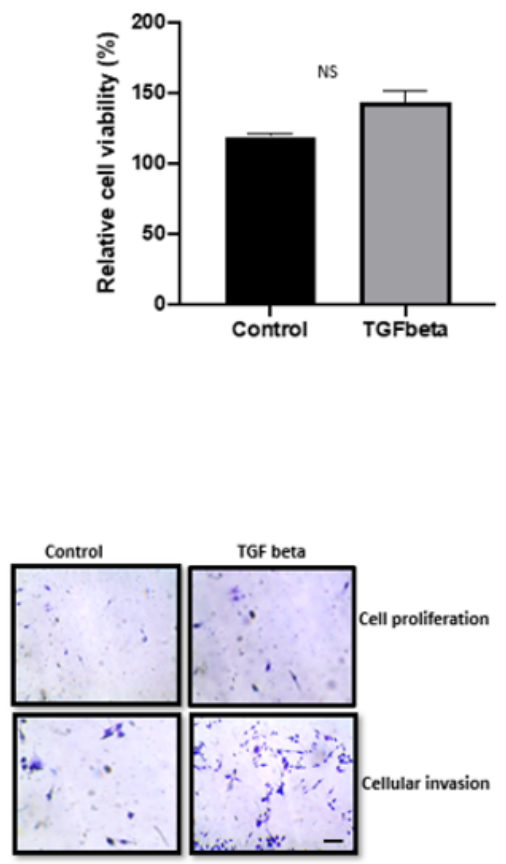

Figure 3: TGF- $\beta$ increased miR-21 expression and invasion of PC3 cells.

A. $\quad$ PC3 cells treated with TGF- $\beta$ induced an elevated expression of miR-21 $(n=6 ; p<0.001)$.

B. TGF- $\beta$ promoted a slight but insignificant increase in the proliferation ability of PC3 cells $(n=3 ; p=0.079)$.

C. A significant increase of PC3 cells invasion capacity was triggered by TGF- $\beta$ ( $n=3 ; p=0.001)$, crystal violet staining was performed to confirmed both cell proliferation and invasion after $16 \mathrm{~h}$. The pictures on the left represent, cell proliferation for control (upper left) and cellular invasion (lower left), the right side of the pictures represent cell proliferation of PC3 cells treated with TGF- $\beta$ (upper right) and cellular invasion (lower right). Scale bar, $20 \mu \mathrm{m}$ (applicable to both panels). 
In order to understand the mechanism behind miR-21 mediated metastatic potential of PC3 prostate cancer cells, we treated cells with $10 \mu \mathrm{M}$ of TGF- $\beta$ for $16 \mathrm{~h}$. TGF- $\beta$ treatment increased miR21 expression 55-fold in PC3 cells (Figure 3A; $n=3 ; p<0.05$ ). In addition, TGF- $\beta$ exposure resulted $175 \%$ increase in PC3 cells invasion capacity over $16 \mathrm{~h}$ (Figure $3 \mathrm{~B} ; \mathrm{n}=6$; $\mathrm{p}<0.05$ ). As expected, there was a slight increase in cell number over the $16 \mathrm{~h}$ but this was insignificant (Figure 3C; $n=6 ; p>0.05$ ). Thus, we concluded that the treatment with TGF- $\beta$ caused a significant increase of miR-21 expression in PC3 cells which led to a higher capacity of invasion and cell proliferation maintaining and/or increasing their metastatic profile.

\section{Discussion}

Increasing clinical and biological evidence showed that a number of miRNAs have potential to monitor disease progression and clarify the molecular mechanism of diseases. miR-21 is an oncogene and found upregulated in six different types of cancer. The upregulated expression level of miR-21 is associated with poor therapeutic response and poor prognosis of disease. Its molecular function is to target several tumour suppressor genes, including programmed cell death 4 (PDCD4) and TGF $\beta R 2$, which are linked to stemness properties of cancer cells [20]. It was shown that miR21 contains a complementary site to $3^{\prime}$ UTR of mRNA of TGF $\beta R 2$, which leads to downregulation of TGF $\beta R 2$. In addition, mir-21 mediated TGF $\beta$ R2 downregulation resulted in a significant increase of c-Myc and Cyclin-D1 expression levels and activated Wnt/ $\beta$ catenin signalling pathway. It is well clarified that TGF $\beta / T G F \beta R 2$ signalling orchestrates crucial cellular processes including cell growth, differentiation, cell death decision, cellular homeostasis and other metabolic cellular functions in the cells.

miR-21 was suggested with a mechanistic role in stemness properties of cells via affecting TGF $\beta$ R2 to modulate $\mathrm{Wnt} / \beta$-catenin signalling [12]. One of the crucial targets of miR-21 is PTEN that often found either deleted or mutated to suppress its function in PCa [6]. Here, we found that PC3 is a PTEN deleted cell line, that showed an increased expression profile for miR-21. In a similar way, VCaP has intact PTEN showed increased expression levels for miR-21 compared to LNCaP and PNT1A cells. PTEN is also the target of miR-221. It was well demonstrated that co-incidence of miR-21 and miR-221 upregulation is a common event in metastatic cancer types such as HCC or OS. Recent findings showed that miR21 showed invasion related PTEN targeting in HCC cells through altering focal adhesion kinase (FAK) phosphorylation and the expression of matrix metalloproteinases MMP2 and MMP9, both downstream mediators of PTEN involved in cell migration and invasion.

We concluded that miR-21 significant upregulation in PC3 cells might be a reason for PTEN deletion and increased the invasion potential of cells through modulating TGF- $\beta$-TIMP signalling axis to regulate MMPs [3]. We found that TGF- $\beta$ upregulated miR-
21 expression in PC3 cells. However, another study showed that upregulation of miR-21 due to TGF- $\beta$ treatment led to growth arrest related to TGFBR2 and PTEN regulation in HaCaT keratinocytes [12]. Thus, the mechanistic role of miR-21 might have potential to regulate the ability of epithelial cells to respond to TGF- $\beta$, with potential impact on epithelium homeostasis, wound-healing and tumorigenesis [12]. According to previous studies, miR21 downregulated Hippo signalling via targeting KIBRA, which promoted the progression of lung adenocarcinoma 28. Hippo signalling axis proteins regulates the miRs biogenesis by activating p72 with YAP to decrease expression levels of miRs in tumour tissues in a cell density dependent manner.

\section{Conclusions}

The role of microRNAs in PCa is increasing its importance. Here, in a panel of PCa cell lines, both mir-21 and miR-221 were seen to be increased agreeing with other studies. We have also looked at potential signalling pathways that miR-21 can regulate. miR-21 may be a diagnostic and prognostic biomarker for PCa. Based on this concept, further research on miRNA signalling pathways are needed.

\section{Acknowledgements}

The authors thank Dr Sigrun Lange for her critical reading.

\section{Funding}

The author(s) received no financial support for the research, authorship, and/or publication of this article.

\section{Declaration of Conflicting Interests}

The author(s) declared no potential conflicts of interest with respect to the research, authorship, and/or publication of this article.

\section{Author Contributions}

Review conception and design: EDA and PUO. Acquisition of data: EDA, ILF, KP, SR, and PUO. Interpretation of data: EDA, ILF, KP and PUO. Drafting the manuscript: EDA, ILF, KP, SR and PUO. Critical revision: EDA and PUO.

\section{References}

1. An Y, Zhang Q, Li X, Wang Z, Li Y, et al. (2018) Upregulated microRNA miR-21 promotes the progression of lung adenocarcinoma through inhibition of KIBRA and the Hippo signaling pathway. Biomedicine and Pharmacotherapy 108: 1845-1855.

2. Camerlingo R, Miceli R, Marra L, Rea G, D’Agnano I, et al. (2019) Conditioned medium of primary lung cancer cells induces EMT in A549 lung cancer cell line by TGF-ß1 and miRNA21 cooperation. PLoS ONE 14(7): e0219597.

3. Dai X, Fang M, Li S, Yan Y, Zhong Y, et al. (2017) miR-21 is involved in transforming growth factor $\beta 1$-induced chemoresistance and invasion by targeting PTEN in breast cancer. Oncology Letters 14(6): 6929-6936.

4. Di Leva G, Garofalo M, Croce CM (2014) MicroRNAs in Cancer. Annual Review of Pathology: Mechanisms of Disease. Annual reviews 9: 287314. 
5. Fendler A, Stephan C, Yousef GM, Jung K (2011) MicroRNAs as regulators of signal transduction in urological tumors. Clinical Chemistry 57(7): 954-968.

6. Fletcher CE, Dart DA, Sita Lumsden A, Cheng H, Rennie PS, et al. (2012) Androgen-regulated processing of the oncome MiR-27a, which targets Prohibiting in prostate cancer. Human Molecular Genetics. 21(14) 3112-3127.

7. Folini M, Gandellini P, Longoni N, Profumo V, Callari M, et al. (2010) miR21: An oncome on strike in prostate cancer. Molecular Cancer 9: 12.

8. Gabriely G, Wurdinger T, Kesari S, Esau CC, Burchard J, et al. (2008) MicroRNA 21 Promotes Glioma Invasion by Targeting Matrix Metalloproteinase Regulators. Molecular and Cellular Biology 28(17): 5369-5380.

9. Garofalo M, Quintavalle C, Romano GM, Croce C, Condorelli G (2011) miR221/222 in Cancer: Their Role in Tumor Progression and Response to Therapy. Current Molecular Medicine 12(1): 27-33.

10. Livak KJ, Schmittgen TD (2001) Analysis of relative gene expression data using real-time quantitative PCR and the $2-\Delta \Delta C$ T method. Methods 25(4): 402-408.

11. Lonergan P, Tindall D (2011) Androgen receptor signalling in prostate cancer development and progression. Journal of Carcinogenesis 10: 20.

12. Magnadóttir B, Uysal Onganer P, Kraev I, Dodds AW, Guðmundsdóttir S, et al. (2020) Extracellular vesicles, deiminated protein cargo and microRNAs are novel serum biomarkers for environmental rearing temperature in Atlantic cod (Gadus morhua L.). Aquaculture Reports 16.

ISSN: 2574-1241

DOI: $10.26717 /$ BJSTR.2020.25.004241

Pinar Uysal-Onganer. Biomed J Sci \& Tech Res

(C) This work is licensed under Creative

Submission Link: https://biomedres.us/submit-manuscript.php
13. Mori M, Triboulet R, Mohseni M, Schlegelmilch K, Shrestha K, et al. (2014) Hippo signalling regulates microprocessor and links cell-densitydependent miRNA biogenesis to cancer. Cell 156(5): 893-906.

14. Nakka M, Allen Rhoades W, Li Y, Kelly AJ, Shen J, et al. (2017) Biomarker significance of plasma and tumor miR-21, miR-221, and miR-106a in osteosarcoma. Oncotarget 8(57): 96738-96752.

15. Perumal D, Leshchenko VV, Kuo PY, Jiang Z, Divakar SKA, et al. (2016) Dual targeting of CDK4 and ARK5 using a novel kinase inhibitor ON123300 exerts potent anticancer activity against multiple myeloma. Cancer Research 76(5): 1225-1236.

16. Siegel RL, Miller KD, Jemal A (2019) Cancer statistics, 2019. CA: A Cancer Journal for Clinicians 69(1): 7-34.

17. Wang T, Zhang L, Shi C, Sun H, Wang J, et al. (2012) TGF- $\beta$-induced miR21 negatively regulates the antiproliferative activity but has no effect on EMT of TGF- $\beta$ in HaCaT cells. International Journal of Biochemistry and Cell Biology 44(2): 366-376.

18. Watahiki A, Wang Y, Morris J, Dennis K, O’Dwyer HM, et al. (2011) MicroRNAs associated with metastatic prostate cancer. PLoS ONE 6(9): e24950.

19. Zaravinos A (2015) The regulatory role of MicroRNAs in EMT and cancer. Journal of Oncology 2015: 865816.

20. Zhang YE (2009) Non-Smad pathways in TGF- $\beta$ signalling. Cell Research 19(1): 128-139.

$\begin{array}{ll}\text { BIOMEDICAL } & \text { Assets of Publishing with us } \\ \text { RESEARCHES } & \text { - Global archiving of articles } \\ & \text { - Immediate, unrestricted online access } \\ & \text { - Rigorous Peer Review Process } \\ & \text { - Anttps://biomedres.us/ }\end{array}$

